\title{
Genetic and Environmental Influences on the Frequency of Orgasm in Women
}

\author{
Khytam Dawood,' Katherine M. Kirk, ${ }^{2}$ J. Michael Bailey, ${ }^{3}$ Paul W. Andrews, ${ }^{4}$ and Nicholas G. Martin ${ }^{2}$ \\ I Department of Psychiatry, University of Chicago, Chicago, Illinois, United States of America \\ 2 Genetic Epidemiology Laboratory, Queensland Institute of Medical Research, Brisbane, Australia \\ 3 Department of Psychology, Northwestern University, Evanston, Illinois, United States of America \\ 4 Virginia Institute of Psychiatric and Behavioral Genetics, Richmond, Virginia, United States of America
}

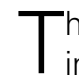
his study reports on genetic and environmental influences on the frequency of orgasm in women during sexual intercourse, during other sexual contact with a partner, and during masturbation. Participants were drawn from the Australian Twin Registry, and recruited from a large, partly longitudinal twin-family study. Three thousand and eighty women responded to the anonymous self-report questionnaire, including 667 complete monozygotic (MZ) pairs and $377 \mathrm{com}-$ plete dizygotic (DZ) same-sex pairs, 366 women from complete DZ opposite-sex pairs, and 626 women whose co-twins did not participate. Significant twin correlations were found for both $\mathrm{MZ}$ and DZ twin pairs for all three items of interest. Age effects were statistically significant for some items. Models incorporating additive genetic, shared and nonshared environmental influences provided the best fit for Items 1 and 3, while a model with additive and nonadditive genetic influences along with nonshared envir-onment fitted the data from Item 2. While an independent pathway model fits the data most par-simoniously, a common pathway model incorporating additive genetic (A), shared environment (C), and unique environment (E) effects cannot be ruled out. Overall, genetic influences account for approximately $31 \%$ of the variance of frequency of orgasm during sexual intercourse, $37 \%$ of the variance of frequency of orgasm during sexual contact other than during intercourse, and $51 \%$ of the variance of frequency of orgasm during masturbation. Following Baker (1996), we speculate that this additive genetic variance might arise from frequency-dependent selection for a variety of female sexual strategies.

Sexual orgasms in women have long been considered a controversial topic (Alzate, 1985), and the study of women's sexual satisfaction in general is extremely complex. Biological and psychosocial factors as varied as hormone levels, personal wellbeing, education levels, and childhood family background have all been demonstrated to play a substantial role in sexual functioning in women (Dennerstein et al., 1999;
Haavio-Mannila \& Kontula, 1997; Raboch \& Raboch, 1992).

In a significant proportion of women, research suggests that other feelings (e.g., physical or emotional closeness to their partner during sexual intercourse) are more important than experiencing orgasm (Busing et al., 2001). Despite this, the inability to achieve orgasm through intercourse is a common sexual complaint (Darling et al., 1991). Ability, frequency and timing of experiencing orgasm have all been demonstrated to have substantial impact on physiological and psychological sexual satisfaction (Darling et al, 1991; Haavio-Mannila \& Kontula, 1997), and the relative infrequency of experiencing orgasm can be felt to be distressing for many women (Raboch \& Raboch, 1992).

With considerable research accessible now on the genetic and environmental etiology of a number of other sexual and reproductive traits in women (Treloar et al., 1999, 2000) it is perhaps surprising that little or no attention has been paid to the possibility of genetic influences on female orgasm. In the present study, we attempt to address this issue by examining self-report questionnaire items specifically pertaining to the frequency of experiencing orgasms in a large, nonclinical sample of female twins.

\section{Method}

\section{Sample}

Twins participating in this study were drawn from the Australian National Health and Medical Research Council Twin Registry, and recruited from two phases of a large, partly longitudinal twin-family study. Questionnaires were mailed to individuals who expressed willingness to participate in an anonymous study of sexual behavior and attitudes. In order to preserve the anonymity of the participants while retaining information on which twins were pairs, twins were

Received 3 November, 2004; accepted 1 December, 2004.

Address for correspondence: Khytam Dawood, PhD, Department of Psychiatry, University of Chicago, 5841 S. Maryland Ave, MC 3077, Chicago, IL 60637, USA. E-mail: khytam@uchicago.edu 
asked to arrange a 10-digit number to serve as a joint identifier with their co-twins and record this number on their questionnaire. Questionnaires were then returned by mail without any other identifiers. Detailed information on the recruitment and administration procedures appears elsewhere (Bailey et al., 2000; Kirk et al., 2000), along with more detailed descriptions of the sample (Dunne et al., 1997).

A total of 1907 complete pairs and 1090 singles returned the questionnaire $54 \%$ of all potential individual participants, and $44 \%$ of all possible twin pairs). Three thousand and eighty women responded to the questionnaire, including 667 complete monozygotic (MZ) pairs and 377 complete dizygotic (DZ) same-sex pairs, 366 women from complete DZ opposite-sex pairs, and 626 women whose co-twins (male or female) did not participate.

\section{Zygosity}

The twins' zygosity was established from responses to standard items about physical similarity, and being mistaken for each other at the time of completion of previous study phases. We and other researchers have shown such items to be at least $95 \%$ accurate when judged against genotyping results (e.g., Kasriel \& Eaves, 1976; Martin \& Martin, 1975; Ooki et al., 1990). The zygosity diagnosis for all individuals was premarked on anonymous questionnaire forms prior to mailing to enable use of this information.

\section{Measures}

Three items pertaining to frequency of experiencing orgasms were included in the questionnaires completed by women: 1) When you have sexual intercourse (i.e., during penetration of the penis), how frequently do you have an orgasm?; 2)How often do you have an orgasm with your sex partner, in ways other than sexual intercourse (e.g., during oral sex)?; and 3) When you masturbate, how frequently do you have an orgasm?

In each case, the response set was: never/rarely (less than $20 \%$ ); fairly often $(20 \%$ to $40 \%)$; often $(40 \%$ to $60 \%)$; usually $(60 \%$ to $80 \%)$; almost always; always; do not do this.

\section{Statistical Methods}

Data were analyzed using SAS 8.00 (SAS Institute, 1999) and Mx 1.50 (Neale, 1999). Correlations between variables are calculated on the assumption that each variable has an underlying continuum of liability which is normally distributed in the population. However, while significant twin correlations establish the fact that there is familial aggregation, they do not distinguish between the possible mechanisms by which this arises. Structural equation modeling is used to make this distinction by considering which combination of additive genetic (A), nonadditive genetic $(D)$, shared environment $(C)$ and unique environment (E) effects provides the most parsimonious explanation for the observed pattern of $\mathrm{MZ}$ and $\mathrm{DZ}$ correlations, subject to the limitation that nonadditive genetic and shared environmental influences are confounded in studies of twins reared together. The extension to multivariate analysis allows the determination of not only sources of covariation but also the pattern or structure in which these differentially influence the covarying measures. The model can then be simplified by determining whether the removal of successive individual parameters results in a significant decrease in the fit of the model to the data. Structural equation modeling was conducted in $\mathrm{Mx} 1.50$ using maximum likelihood methods for raw ordinal data.

\section{Results}

\section{Response Frequencies}

The category response percentages for each of the three measures of frequency of orgasm in women are shown in Table 1. Different category endorsement frequencies were observed for each of the three measures $(p<$ .001 ), even after accounting for different proportions of women experiencing each of the situations.

In the analyses outlined below, responses in the final category for each item ('have never had intercourse/do not do this') were treated as missing values. No significant differences in the distribution of responses were observed between women in same-sex and opposite-sex twin pairs where both twins participated in the study $(p=.493$ for Item $1, p=.386$ for Item $2, p=.878$ for Item 3 ), nor was there a significant difference between these response frequencies and those from women whose co-twins did not take part in the study ( $p=.101, p=.360, p=.671$ respectively). Thresholds for women in same-sex and opposite-sex twin groups and between complete and incomplete pairs were constrained equal in all models fitted.

\section{Univariate Analysis}

Significant twin correlations were found for both $\mathrm{MZ}$ and $\mathrm{DZ}$ twin pairs for all three items of interest. Age effects were corrected for, and found to be statistically significant for, Items 1 and 3; older women endorsed the categories at the lower end of the scale more frequently than younger women for these items. Responses between MZ co-twins correlated $.31, .38$ and .54 for the items on orgasm frequency during sexual intercourse, sexual interaction in ways other than intercourse, and masturbation, while the corresponding DZ twin correlations were .16,.14 and .34 respectively.

Univariate structural equation modeling results are shown in Table 2. As would be expected from the correlation values above, models incorporating additive genetic and shared and nonshared environmental influences provided the best fit for Items 1 and 3, while a model with additive and nonadditive genetic influences along with nonshared environment fitted the data from Item 2. In each case, genetic influences were statistically significant, although the shared environmental effects (Items 1 and 3) or nonadditive genetic effects 


\section{Table 1}

Category Response Percentages for Measures of Frequency of Orgasm in Women

\begin{tabular}{lcccccccrrr}
\hline & \multicolumn{1}{c}{ Response categories } \\
& $N$ & Never & $\begin{array}{c}\text { Rarely } \\
(<20 \%)\end{array}$ & $\begin{array}{c}\text { Fairly often } \\
(20-40 \%)\end{array}$ & $\begin{array}{c}\text { Often } \\
(40-60 \%)\end{array}$ & $\begin{array}{c}\text { Usually } \\
(60-80 \%)\end{array}$ & $\begin{array}{r}\text { Almost } \\
\text { always }\end{array}$ & Always & Do not do \\
\hline Sexual intercourse & 2901 & 13.7 & 21.0 & 14.0 & 11.5 & 13.1 & 17.9 & 5.3 & 3.7 \\
With partner, other than intercourse & 2896 & 13.6 & 19.8 & 13.5 & 10.0 & 11.3 & 16.5 & 10.4 & 5.0 \\
Masturbation & 2896 & 10.8 & 7.0 & 4.5 & 3.9 & 4.7 & 10.7 & 27.2 & 31.1 \\
\hline
\end{tabular}

(Item 2) could be removed from the model without a significant decrease in goodness-of-fit.

\section{Multivariate Analysis}

Cross-twin cross-trait correlations for MZ and DZ twin pairs are shown in Table 3 . As found in the univariate analysis, the correlations for $M Z$ twin pairs are greater than those for DZ twin pairs. Further inspection of Table 3 reveals that in several cases the cross-trait cross-twin correlations for $\mathrm{MZ}$ twin pairs is more than twice the corresponding DZ twin correlation (potentially indicating nonadditive genetic influences), while in other cases the MZ twin correlation is less than twice the DZ correlation (suggesting shared environmental effects). Even though these deviations were nonsignificant in the univariate analyses, both a full ACE Cholesky model and a full ADE Cholesky model were fitted to the data as starting points for the multivariate modeling process. The model containing shared environmental influences (ACE) fitted the data slightly better than the model incorporating nonadditive genetic influences (ADE) $(\Delta[-2 \mathrm{LL}]=1.695)$. However, the shared environmental effects in the ACE model were not statistically significant, and the model was able to be reduced to incorporate only additive genetic effects and nonshared environment (an AE model) without significant loss of fit $\left(\Delta \chi_{6}^{2}=3.219, p=.781\right)$.

In order to test the hypothesis that there are genetic and environmental influences common to the three observed variables, we applied an independent pathway model (Kendler et al., 1987) to the data. Since neither shared environmental nor nonadditive genetic effects had previously been found to be statistically significant, the independent pathway model incorporated only additive genetic and nonshared environmental influences. Comparison of the fit of this independent pathway model to that of the full ACE Cholesky model (above) revealed no significant loss of fit $\left(\Delta \chi_{6}^{2}=3.230\right)$. The genetic and environmental influences common to the three observed variables account for different proportions of the three variables contributing between $9 \%$ and $65 \%$ of the variance as shown in Figure 1. Overall, genetic influences account for approximately $31 \%$ of the variance of frequency of orgasm during sexual intercourse, $37 \%$ of the variance of frequency of orgasm during sexual contact with a partner other than during intercourse and $51 \%$ of the variance of frequency of orgasm during masturbation.

A common pathway model (Kendler et al., 1987) was also applied to the data in order to test the more restrictive hypothesis that a common latent construct underlies the three observed variables. In contrast to the independent pathway model, a common pathway model incorporating only additive genetic and nonshared environmental effects did not provide a good fit to the data relative to the full ACE Cholesky model $\left(\Delta \chi^{2}{ }_{7}=89.592, p=.000\right)$, and could thus be rejected. However, the addition of shared environmental influences specific to the variables relating to frequency of orgasm during sexual intercourse and masturbation (as per Figure 2) improved the fit substantially. Compared to the independent pathway model presented in Figure 1, this common pathway has slightly

Table 2

Results of Univariate Structural Equation Modeling with 95\% Confidence Intervals

\begin{tabular}{|c|c|c|c|c|c|}
\hline & A & D & $A+D$ & C & $\mathrm{E}$ \\
\hline Sexual intercourse & $\begin{array}{c}.29 \\
(.02-.38)\end{array}$ & - & $\begin{array}{c}.29 \\
(.02-.39)\end{array}$ & $\begin{array}{c}.02 \\
(.00-.25)\end{array}$ & $\begin{array}{c}.69 \\
(.62-.77)\end{array}$ \\
\hline With partner, other than intercourse & $\begin{array}{c}.20 \\
(.00-.43)\end{array}$ & $\begin{array}{c}.18 \\
(.00-.45)\end{array}$ & $\begin{array}{c}.38 \\
(.30-.45)\end{array}$ & - & $\begin{array}{c}.62 \\
(.55-.70)\end{array}$ \\
\hline Masturbation & $\begin{array}{c}.40 \\
(.02-.62)\end{array}$ & - & $\begin{array}{c}.40 \\
(.02-.63)\end{array}$ & $\begin{array}{c}.14 \\
(.00-.47)\end{array}$ & $\begin{array}{c}.47 \\
(.37-.57)\end{array}$ \\
\hline
\end{tabular}

Note: Additive genetic, nonadditive genetic, shared environmental and nonshared environmental influences are represented by $A, D, C$ and $E$ respectively. The estimate of the total genetic influence on a measure is given by $A+D$. 
Table 3

Cross-Twin, Cross-Trait Correlations for Variables Related to Frequency of Having an Orgasm During Sexual Intercourse, During Sexual Interaction with a Partner in Ways Other Than Intercourse, and During Masturbation

\begin{tabular}{|c|c|c|c|c|c|c|}
\hline & \multicolumn{3}{|c|}{ Twin 1} & \multicolumn{3}{|c|}{ Twin 2} \\
\hline & Intercourse & $\begin{array}{l}\text { With partner, } \\
\text { other than } \\
\text { intercourse }\end{array}$ & Masturbation & Intercourse & $\begin{array}{c}\text { With partner, } \\
\text { other than } \\
\text { intercourse }\end{array}$ & Masturbation \\
\hline \multicolumn{7}{|l|}{ Twin 1} \\
\hline Sexual intercourse & 1.00 & .28 & .24 & .31 & .19 & .18 \\
\hline With partner, other than intercourse & .15 & 1.00 & .50 & .12 & .38 & .28 \\
\hline Masturbation & .07 & .49 & 1.00 & .09 & .29 & .49 \\
\hline \multicolumn{7}{|l|}{ Twin 2} \\
\hline Sexual intercourse & .17 & .04 & .03 & 1.00 & .19 & .19 \\
\hline With partner, other than intercourse & -.03 & .14 & .12 & .31 & 1.00 & .54 \\
\hline Masturbation & -.05 & .14 & .32 & .21 & .47 & 1.00 \\
\hline
\end{tabular}

Note: Monozygotic (MZ) twin results are above the main diagonal, with dizygotic (DZ) correlations below the main diagonal. Correlations between twins for individual traits (as estimated in the multivariate analysis) are shown in bold.

better fit to the data, but is less parsimonious $(\Delta-2 \log$ likelihood $=-0.768, \Delta d f=3$ ).

\section{$\overline{\text { Discussion }}$}

Results from the current study indicate that the independent pathway AE model fits the data most parsimoniously (using AIC criterion). However, a common pathway model incorporating $\mathrm{A}, \mathrm{C}$ and $\mathrm{E}$ effects cannot be ruled out. Further, the common or shared environment effects found in the present study may well reflect the childhood family background (sexually uninhibited, nonreligious; Haavio-Mannila \& Kontula, 1997).

Results also indicate that the frequency of orgasms experienced were different for each type of sexual activity with higher frequency reported during sexual intercourse, or 'with a partner, other than intercourse'. These findings appear to support results from previous studies reporting that partner involvement was a noted preference for many women (Darling et al., 1991; Davidson \& Darling, 1989).

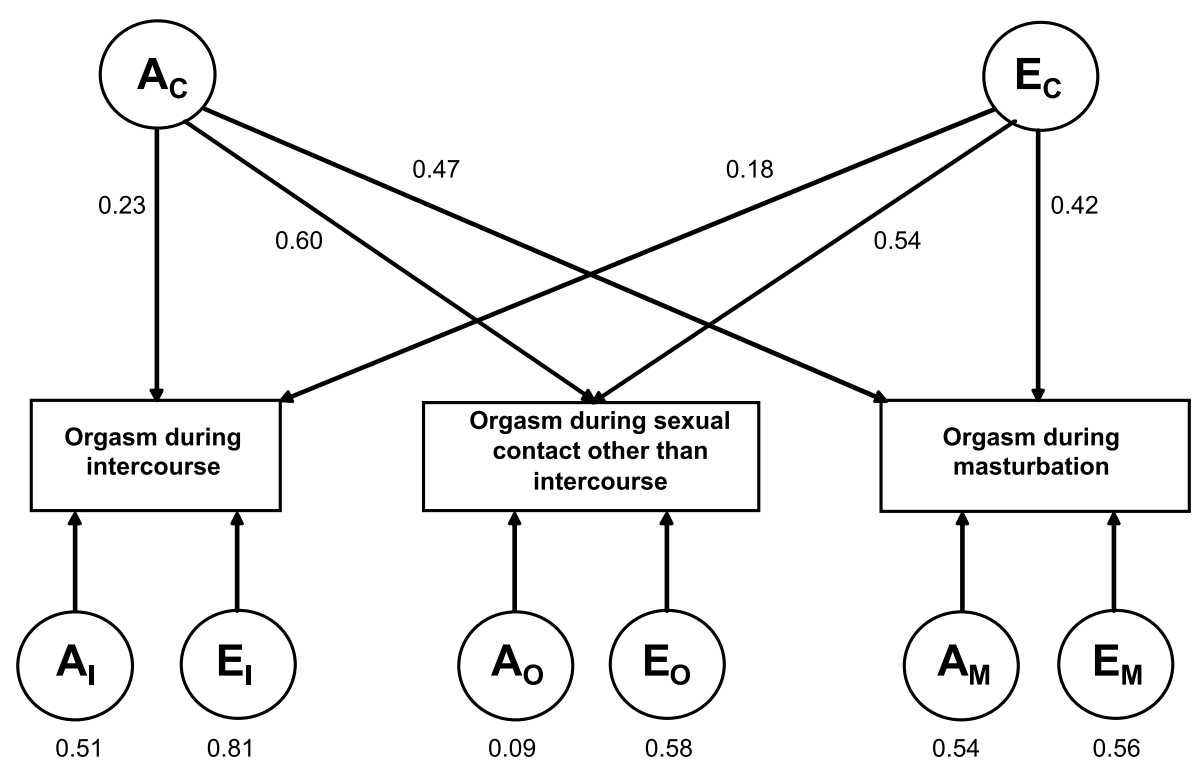

\section{Figure 1}

Path diagram of independent pathway model showing latent genetic and environmental influences on the measured phenotypes of frequency of experience of orgasm during sexual intercourse, during sexual contact with a partner other than intercourse, and during masturbation.

Note: $A_{C}$ and $E_{c}$ represent additive genetic and environmental factors common to all three variables, while $A_{1}, A_{0}$ and $A_{M}$ and $E_{1}, E_{0}$ and $E_{M}$ represent additive genetic and environmental factors specific to the individual variables. Path coefficients are shown. These coefficients must be squared to obtain the proportions of variance of each measured variable accounted for by the latent variable. 


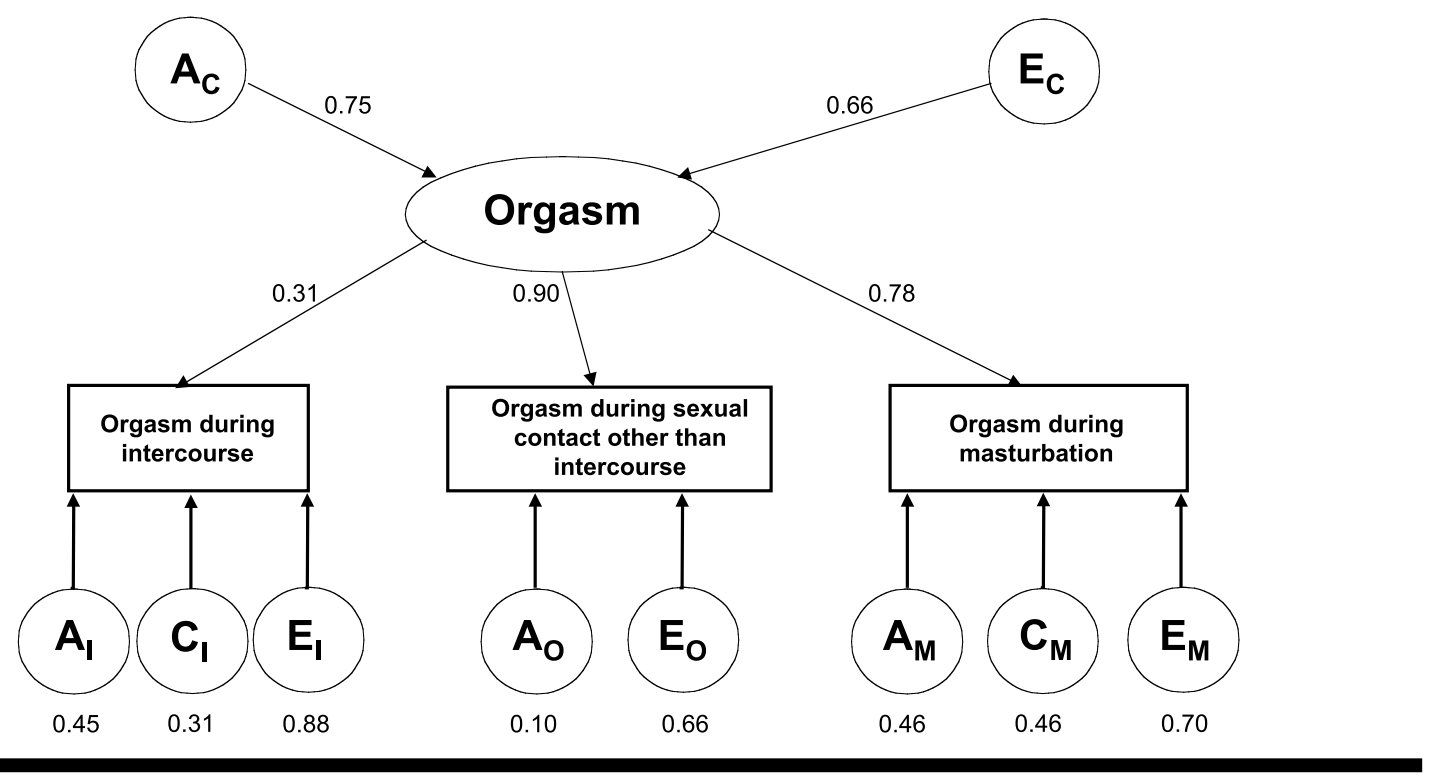

\section{Figure 2}

Path diagram of common pathway model showing latent genetic and environmental influences on the measured phenotypes of frequency of experience of orgasm during sexual intercourse, during sexual contact with a partner other than intercourse, and during masturbation.

Note: $A_{c}$ and $E_{c}$ represent additive genetic and environmental factors common to all three variables, while $A_{1}, A_{0}$ and $A_{m}, C_{1}$ and $C_{m}$, and $E_{l}, E_{0}$ and $E_{m}$ represent additive genetic, shared environmental and nonshared environmental factors specific to the individual variables. Path coefficients are shown. These coefficients must be squared to obtain the proportions of variance of each measured variable accounted for by the latent variable.

Analysis of the anonymous item responses of twins whose co-twin responded to the survey versus those whose co-twin did not respond can provide an estimate of the volunteer biases affecting those individual items provided that the same encouragement to participate has been given to individuals regardless of the participation status of their relatives (Neale \& Eaves, 1993). This analysis, applied to the three items relating to frequency of experiencing orgasm, found no evidence of a significant volunteer bias for any of the three measures, which would tend to indicate that this type of volunteer bias has not significantly affected the results of the present study.

Setting the 'do not do' category to missing for the masturbation item results in deletion of $30 \%$ of the sample and this presents a potential problem of ascertainment bias. Ideally one should use a two-stage model as used by Heath et al. (2002) for number of cigarettes which cannot be scored if participants have never smoked. But imputation of missing values in multivariate analysis allows partial correction for this as the number missing for the other two items is very much lower. Interestingly, the heritabilities of all three orgasm items from the multivariate ACE Cholesky model are all slightly lower $(.22, .33$ and .32 , results not shown) than their univariate counterparts (.29, .38 and .40 , Table 2 ), suggesting there may be some ascertainment bias affecting the univariate results.

Given the relative dearth of studies conducted on the possibility of genetic influences on the frequency of orgasm in women, our findings present an important contribution to the literature and may have substantial implications for future research in this area.

Various functional hypotheses for female orgasm have been proposed (Buss, 2003; Levin, 2002). There is some evidence that female orgasm helps maintain vaginal and pelvic functionality (Levin, 2003). Another hypothesis proposes that female orgasm is a selective sperm-retention device (Baker \& Bellis, 1993). This hypothesis utilizes the fact that female orgasm reverses the pressure differential in the uterus and causes an 'upsuck' effect (Fox, 1976), which may help reduce 'flowback' and enhance the chances of fertilization (Baker \& Bellis, 1993). The hypothesis suggests that some of the variation in women's reported frequency of orgasm is related to differences in male quality for siring offspring. Some support is found in a study in which women were more likely to report orgasm when they were paired with men who scored higher on an index thought to reflect genetic quality - bilateral symmetry (Thornhill et al., 1995).

Many evolutionary psychologists believe that species- or sex-typical adaptations should exhibit little genetic variation because selection should winnow out the deleterious mutants that impair functioning. However, traits whose phenotypes depend on a large part of the genome (e.g., intellectual ability) can show a substantial amount of genetic variation due to mutational load (Miller, 2000; Rowe \& Houle, 1996). For instance, if thousands of genes code for the trait, mutation could introduce substantial variation in the trait even if selection is winnowing out deleterious mutations. Moreover, if there is assorta- 
tive mating on the basis of the trait, it will exacerbate the variation in the population.

Even if female orgasm is an adaptation with little genetic variation, a twin study could still find that there is substantial genetic variation correlated with the expression of the phenotype. In the absence of a specific model, a twin study is correlational and does not specify the pathway from genotype to phenotype. For instance, our study is unable to rule out the possibility that the genetic variation associated with the reported frequency of orgasm could actually reflect variation in women's ability to pair-bond with males who would make good sires for their offspring.

While our results give us little traction on specific evolutionary hypotheses, they do suggest that orgasm during intercourse, orgasm during sexual contact other than intercourse and orgasm during masturbation may be distinct phenotypes. This is supported both by the failure of the common pathway model to provide the best fit, and by the significant influence of the contextspecific genetic and environmental effects. In other words, female orgasm may function differently in different contexts. For future work attempting to elucidate the evolved functions of female orgasm, it may be important not to generalize findings from one context to another.

Nevertheless, our findings suggest that there may be wide, genetically based variety between females in their sexual responses and reproductive strategies. Baker (1996, chapter 11) suggests that there are four broad categories of sexual response in women: (i) about $75 \%$ of women who are programmed sometimes to have (and sometimes not to have) the full range of possible orgasms (masturbatory, nocturnal, foreplay, intercourse, and postplay - with some being multiple); (ii) about $10 \%$ of women who either do not have masturbatory orgasms or do not climax in the presence of men; (iii) about $10 \%$ of women who climax virtually every time they have penetrative sex; and (iv) about $2 \%$ to $4 \%$ of women who never have an orgasm. Baker argues that female orgasm has generally evolved to selectively retain sperm and manipulate competition between sperm from inseminations by different men. But he also argues that it could be advantageous for women to be different from each other in their sexual responsiveness (e.g., to ensure insemination by a highly skilled partner, to avoid pair-bonding to a male who is unlikely to invest in offspring, and so forth). If these categories of sexual responsiveness have different strengths and weaknesses in maintaining sperm competition and inducing partner fidelity, they could be maintained by frequency-dependent selection which will maintain genetic variance for component phenotypes, including orgasm frequency. One test of Baker's hypothesis would involve latent class analysis to see if we can recover his predicted classes, followed by genetic analysis of class probabilities, which we plan to do.

\section{Acknowledgments}

This work was funded by grants to JMB (NIMH) and NGM (NHMRC - CARG). We thank the staff of the Genetic Epidemiology Unit at the Queensland Institute of Medical Research for assistance and the twins for their cooperation.

\section{References}

Alzate, H. (1985). Vaginal eroticism and female orgasm: A current appraisal. Journal of Sex and Marital Therapy, 11, 271-284.

Bailey, J. M., Dunne, M. P., \& Martin, N. G. (2000). The distribution, correlates and determinants of sexual orientation in an Australian twin sample. Journal of Personality and Social Psychology, 78, 524-536.

Baker, R. R. (1996). Sperm wars: The science of sex. New York: HarperCollins

Baker, R. R., \& Bellis, M. A. (1993). Human sperm competition: Ejaculate manipulation by females and a function for the female orgasm. Animal Behaviour, 46, 887-909.

Busing, S., Hoppe, C., \& Liedke, R. (2001). Sexual satisfaction of women: Development and results of a questionnaire. Psychotherapie, Psychosomatik, Medizinische Psychologie, 51, 68-75.

Buss, D. M. (2003). The evolution of desire: Strategies of human mating. New York: BasicBooks.

Darling, C. A., Davidson, J. K., Sr., \& Jennings, D. A. (1991). The female sexual response revisited: Understanding the multiorgasmic experience in women. Archives of Sexual Behavior, 20, 527-540.

Davidson, J. K., \& Darling, C. A. (1989). Self-perceived differences in the female orgasmic response. Family Practice Research Journal, 8, 75-84.

Dennerstein, L., Lehert, P., Burger, H., \& Dudley, E. (1999). Factors affecting sexual functioning of women in the mid-life years. Climacteric, 2, 254-262.

Dunne, M. P., Martin, N. G., Bailey, J. M., Heath, A. C., Bucholz, K. K., Madden, P. A. F., \& Statham, D. J. (1997). Participation bias in a sexuality survey: Psychological and behavioural characteristics of responders and non-responders. International Journal of Epidemiology, 26, 844-854.

Fox, C. A. (1976). Some aspects and implications of coital physiology. Journal of Sex and Marital Therapy, 2, 205-213.

Haavio-Mannila, E., \& Kontula, O. (1997). Correlates of increased sexual satisfaction. Archives of Sexual Behavior, 26, 399-419.

Heath, A. C., Martin, N. G., Lynskey, M. J., Todorov, A. A., \& Madden, P. A. F. (2002). Estimating two-stage models for genetic influences on alcohol, tobacco or drug use initiation and dependence vulnerability in twin and family data. Twin Research, 5, 113-124. 
Kasriel, J., \& Eaves, L. J. (1976). A comparison of the accuracy of written questionnaires with blood typing for diagnosing zygosity in twins. Journal of Biosocial Science, 8, 263-266.

Kendler, K. S., Heath, A. C., Martin, N. G., \& Eaves, L. J. (1987). Symptoms of anxiety and symptoms of depression. Same genes, different environments? Archives of General Psychiatry, 44, 451-457.

Kirk, K. M., Bailey, J. M., Dunne, M. P., \& Martin, N. G. (2000). Measurement models for sexual orientation in a community twin sample. Behavior Genetics, 30, 345-356.

Levin, R. J. (2002). The physiology of sexual arousal in the human female: A recreational and procreational synthesis. Archives of Sexual Behavior, 31, 405-441.

Levin, R. J. (2003). Do women gain anything from coitus apart from pregnancy? Changes in the human female genital tract activated by coitus. Journal of Sex and Marital Therapy, 29(Suppl. 1), 59-69.

Martin, N. G., \& Martin, P. G. (1975). The inheritance of scholastic abilities in a sample of twins: I. Ascertainment of the sample and diagnosis of zygosity. Annals of Human Genetics, 39, 213-218.

Miller, G. F. (2000). The mating mind: How sexual choice shaped the evolution of human nature. New York: Doubleday.

Neale, M. C. (1999). Mx: Statistical modeling (4th ed.). Richmond, VA: Department of Psychiatry, Medical College of Virginia.
Neale, M. C., \& Eaves, L. J. (1993). Estimating and controlling for the effects of volunteer bias with pairs of relatives. Behavior Genetics, 23, 271-277.

Ooki, S., Yamada, K., Asaka, A., \& Hayakawa, K. (1990). Zygosity diagnosis of twins by questionnaire. Acta Geneticae Medicae et Gemellologiae, 39, 109-115.

Raboch, J., \& Raboch, J. (1992). Infrequent orgasms in women. Journal of Sex and Marital Therapy, 18, 114-120.

Rowe, L., \& Houle, D. (1996). The lek paradox and the capture of genetic variance by condition dependent traits. Proceedings of the Royal Society of London. Series B. Biological Sciences, 263, 1415-1421.

SAS. (1999). SAS (Version 8). Carey, NC: SAS Institute.

Thornhill, R., Gangestad, S. W., \& Comer, R. (1995). Human female orgasm and mate fluctuating asymmetry. Animal Behavior, 50, 1601-1615.

Treloar, S. A., Martin, N. G., Bucholz, K. K., Madden, P. A., \& Heath, A. C. (1999). Genetic influences on postnatal depressive symptoms: Findings from an Australian twin sample. Psychological Medicine, 29, 645-654.

Treloar, S. A., Macones, G. A., Mitchell, L. E., \& Martin, N. G. (2000). Genetic influences on premature parturition in an Australian twin sample. Twin Research, 3, 80-82. 